\title{
Karl Bartsch, Gaston Paris, Correspondance, U. Bähler (éd.)
}

\section{Germana Berlantini}

\section{(2) OpenEdition}

1 Journals

\section{Edizione digitale}

URL: http://journals.openedition.org/studifrancesi/10101

DOI: 10.4000/studifrancesi.10101

ISSN: 2421-5856

\section{Editore}

Rosenberg \& Sellier

\section{Edizione cartacea}

Data di pubblicazione: 1 août 2017

Paginazione: 421-422

ISSN: 0039-2944

\section{Notizia bibliografica digitale}

Germana Berlantini, «Karl Bartsch, Gaston Paris, Correspondance, U. Bähler (éd.)», Studi Francesi [Online], 182 (LXI | II) | 2017, online dal 01 août 2017, consultato il 06 janvier 2021. URL: http:// journals.openedition.org/studifrancesi/10101; DOI: https://doi.org/10.4000/studifrancesi.10101

Questo documento è stato generato automaticamente il 6 janvier 2021.

\section{(c) (i) $\odot$}

Studi Francesi è distribuita con Licenza Creative Commons Attribuzione - Non commerciale - Non opere derivate 4.0 Internazionale. 


\title{
Karl Bartsch, Gaston Paris, Correspondance, U. Bähler (éd.)
}

\author{
Germana Berlantini
}

\section{NOTIZIA}

Karl Bartsch, Gaston Paris, Correspondance. Entièrement revue et complétée par Ursula BÄHLER à partir de l'édition de Mario ROQUES, Firenze, Edizioni del Galluzzo per la fondazione Ezio Franceschini, 2015, 136 pp.

1 Secondo volume della collezione «L'Europe des philologues. Correspondances», la corrispondenza fra Karl Bartsch e Gaston Paris copre l'arco ventennale di un'amicizia al crocevia fra relazione profondamente umana e intenso dialogo scientifico e culturale. Un'amicizia che, a eccezione di alcuni rari incontri de visu si era prevalentemente sviluppata grazie a uno scambio epistolare protrattosi dal 1865 al 1885. Grazie alla solerzia della vedova Paris, di Joseph Bédier e dello stesso Mario Roques, dopo la morte dello studioso quest'ultimo aveva potuto raccogliere tutta la documentazione.

Roques aveva progettato di suddividerne la pubblicazione in quattro parti, delle quali solo tre videro la luce nell'arco della sua vita: le lettere degli anni 1865-1867 pubblicate nel 1927 nel volume Medieval Studies in Memory of Gertrude Schoepperle Loomis; quelle del periodo 1868-1870 edite nel 1931 nel periodico «Neuphilologische Mitteilungen» e infine la corrispondenza del 1871 nel contesto del volume A Miscellany of Studies in Romance Languages \& Literatures, presented to Leon E. Kastner apparso nel 1932. Una quarta parte con ogni probabilità venne lasciata inedita da Roques in quanto recante traccia dell'"affaire Kutschke", polemica che Paul Meyer aveva istruito contro Karl Bartsch a proposito della collaborazione di quest'ultimo alla pubblicazione di un testo parodico anti-napoleonico da parte di Franz Wilhelm Ehrenthal. Avendo Gaston Paris preso posizione in difesa di Bartsch e esplicitamente criticato Mayer, Roques con ogni probabilità ha giudicato opportuno proteggere l'immagine di quest'ultimo. Ursula BÄHLER - che nella prefazione sottolinea come i toni pur accesi di Mayer non 
corrispondessero ad alcuna forma di sciovinismo da parte sua e che anzi egli insieme a Paris aveva preso posizione durante l'Affaire Dreyfus a sostegno della sua innocenza ne ha curato una prima pubblicazione nel 2003 su «Romania». La riedizione per i tipi delle Edizioni del Galluzzo, non solo riunisce tutte le lettere editate nelle quattro versioni precedenti, ma reintegra i passaggi più personali e autobiografici omessi da Roques e che per il lettore contemporaneo costituiscono una testimonianza rilevante tanto sull'amicizia umana e scientifica che univa i due studiosi quanto sulla personalità di ciascuno di loro.

Ursula Bähler traccia i profili biografici e scientifici dei due filologi nell'introduzione al volume. Karl Bartsch proveniva da studi di filologia classica e di letteratura tedesca, tanto moderna quanto medioevale, ed era approdato da autodidatta allo studio della langue d'oc e della langue d'oil nel cui campo era destinato ad eccellere. Ordinario di filologia germanica e romanza all'università di Heidelberg, Bartsch era uno degli studiosi più prolifici della sua generazione, fra i pochi a portare avanti entrambe le branche della disciplina (all'epoca già abbastanza specializzate e diversificate) oltre che a coniugare interessi scientifici e pedagogici, secondo un orientamento che lo aveva visto opporsi, al fianco di Franz Pfeiffer, Adolf Holtzmann, Friedrich Zarncke, ai "lachmaniani" berlinesi, accusati di praticare una filologia élitaria. Nondimeno è ai principi di edizione di Lachmann che Bartsch si rifà per le sue edizioni critiche trasferendoli su testi francesi e provenzali medioevali cui affianca la pubblicazione di manuali per i professori e per gli studenti.

4 Gaston Paris proveniva invece da studi di diritto e contemporaneamente si era diplomato come archivista-paleografo all'École des chartes. Alla successione al padre al Collège de France, aggiunge nel 1896 la nomina all'Académie française. Il suo nome è legato alla fondazione del periodico «Romania» insieme a Paul Meyer oltre che a quella della "Société des Anciens Textes Français". Accanto alla monumentale Histoire poétique de Charlemagne bisogna menzionare fra i suoi lavori almeno La Littérature française au Moyen Âge e il libro consacrato a François Villon.

Lo scambio epistolare fra Bartsch e Paris costituisce una preziosa testimonianza storica del lavoro filologico in un'epoca in cui la dipendenza reciproca degli studiosi era di gran lunga maggiore rispetto ai giorni nostri e in cui copiare e collazionare testi e manoscritti o inviarsi reciprocamente libri era pratica comune e necessaria. Nelle lettere non mancano neppure le discussioni su questioni di metrica o di edizione; commenti sulle differenti pratiche filologiche dei due paesi; critiche reciproche di grande franchezza. È tuttavia all'indomani della morte di Bartsch che Paris esplicita, pur entro la generale ammirazione per il lavoro del collega tedesco, una distanza critica nei confronti di una certa arditezza, in linea col più generale dubbio di amatorialità che colpiva in particolar modo le sue ricerche linguistiche. La stessa Bähler, a conclusione della sua introduzione, sottolinea la portata ideologica di questo gesto col quale Paris afferma la maturità e modernità scientifica della filologia francese, pienamente iscritta nel paradigma storico-comparativo.

6 Il volume si compone, oltre che dell'introduzione di Ursula Bähler (pp. VII-XLI), di una sezione intitolata «Documents», concernente le fonti e i criteri d'edizione (pp. XLIII$\mathrm{XLVI}$ ), della corrispondenza fra i due studiosi, riprodotta in lingua originale (tedesco e francese, senza traduzioni; pp. 3-120), di un'«Annexe» fotografica (pp. 121-126) e dell'indice dei nomi (pp. 127-136). 